\title{
Cisticercose suína e bovina - ocorrência em abatedouros do Estado de São Paulo, Brasil*
}

\section{Swine and bovine cysticercosis - occurrence in slaughterhouses located at São Paulo State, Brazil}

\author{
Gabriel Augusto Marques Rossi, ${ }^{* *}$ Maria Fernanda Garnica, ${ }^{* *}$ Henrique Meiroz de Souza Almeida, ${ }^{* *}$ Laryssa Freitas Ribeiro, ${ }^{* *}$ \\ Ana Maria Centola Vidal Martins, ${ }^{* * *}$ Karina Paes Bürger, ${ }^{* *}$ Luiz Francisco Prata, ${ }^{* *}$ Luis Antonio Mathias*
}

\begin{abstract}
Resumo
Objetivou-se determinar a frequência de ocorrência da cisticercose em carcaças suínas e bovinas em 56 abatedouros sob fiscalização do Serviço de Inspeção do Estado de São Paulo (SISP) durante os anos 2008 a 2013. Para os 1.305 .723 suínos abatidos no período foram detectados cisticercos em apenas $125(0,01 \%)$ animais, dos quais $116(92,80 \%)$ estavam calcificados. Determinou-se que dos 1.434 .445 bovinos, $24.278(1,69 \%)$ apresentaram cisticercos, dos quais 19.826 (81,67\%) apresentavamse calcificados. Também se observou uma relação inversa $(P<0,05)$ entre as variáveis tempo e frequência de ocorrência. $A$ maior ocorrência nessa espécie evidencia que são necessárias melhorias nas práticas sanitárias preventivas durante a criação desses animais, como a adoção das Boas Práticas Agropecuárias (BPA) para reduzir a ocorrência da cisticercose a níveis baixos igualmente observados para os suínos.
\end{abstract}

Palavras-chave: Taenia saginata, Taenia solium, Zoonoses.

\begin{abstract}
This study was focused on the determination of cysticercosis prevalence in swine and cattle carcasses in 56 slaughterhouses under supervision from Inspection Service of São Paulo State (SISP) between the years 2008 and 2013. Amongst the 1,305,723 pigs slaughtered, cysticercosis was present in only $125(0.01 \%)$ of them in which, $116(92.80 \%)$ were calcified. Nonetheless, cysticercosis was present in $24,278(1.69 \%)$ of the $1,434,445$ bovine carcasses evaluated; $19,826(81.67 \%)$ were calcified. An inverse relationship $(\mathrm{P}<0.05)$ between time and the disease occurrence variable was observed. Sanitary preventive practices improvements, such as the Good Agricultural Practices, are required during the cattle rearing in order to decrease the cysticercosis occurrence to low prevalence levels as observed in swine rearing.
\end{abstract}

Keywords: Taenia saginata, Taenia solium, Zoonosis

\section{Introdução}

O complexo teniose-cisticercose suíno e bovino é uma zoonose causada pelos cestódeos Taenia solium e T. saginata, respectivamente. A teniose consiste na ocorrência da enfermidade pela presença da forma adulta dos parasitas nos humanos, os quais são hospedeiros definitivos para ambas as espécies e se infectam ao ingerirem cisticercos viáveis. Já a cisticercose é causada pelas formas larvares desses mesmos parasitas, que possuem como hospedeiros intermediários os bovinos no caso da $T$. saginata e os suínos e humanos no caso da $T$. solium, que se infectam ao ingerirem água ou alimentos contaminados com ovos do parasita (Pfuetzenreiter e Ávila-Pires, 2000; Santos e Barros, 2009).

A inspeção sanitária das carnes destaca-se como uma das medidas efetivas para o controle do complexo teniosecisticercose. Consiste em fonte de informação aos serviços de saúde pública e animal por disponibilizar dados de ocorrência através dos registros de casos detectados durante a inspeção post-mortem. Também permite adequada destinação para cada animal parasitado de acordo com a quantidade de cisticercos e forma dos mesmos, que podem estar vivos ou calcificados (Pinto, 2008). As destinações utilizadas são o comprometimento da exportação, tratamento térmico pelo frio ou calor ou a condenação total (Brasil, 1952), o que acarreta em consideráveis prejuízos para todos os elos da cadeia produtiva da carne brasileira.

Diante desse cenário, objetivou-se verificar a ocorrência da cisticercose em carcaças suínas e bovinas em 56 abatedouros sob fiscalização do Serviço de Inspeção do Estado de São Paulo (SISP) durante os anos 2008 a 2013.

\section{Material e métodos}

Foram utilizados dados de relatórios do SISP referentes à ocorrência de cisticercose (viva e calcificada) em 56 abatedouros

\footnotetext{
*Recebido em 6 de outubro de 2014 e aceito em 20 de setembro de 2015.

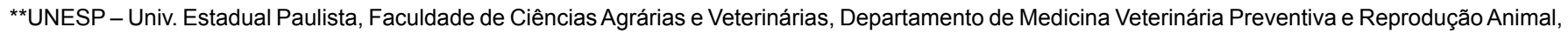
Via de Acesso Prof, Paulo Donatto Castellane s/n, Jaboticabal/SP, Brasil, Cep 14884-900.

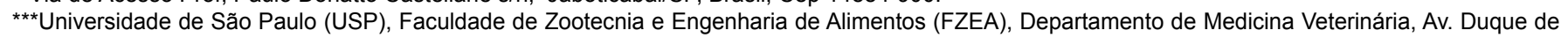
Caxias Norte 225, Pirassununga/SP, Brasil, Cep 13635-900.

Autor para correspondência: gabrielrossiveterinario@gmail.com
} 
que realizavam o abate de suínos e bovinos durante os anos de 2008 a junho de 2013. Nas planilhas consultadas, constavam o número de registro da indústria, a espécie abatida, os números mensais de animais e de casos de cisticercose viva e calcificada.

A análise estatística de regressão linear dos dados para bovinos foi realizada através do Software $\mathrm{R}^{\circledR}$ utilizando o nível de $5 \%$ de significância $(P<0,05)$.

\section{Resultados e discussão}

Durante o período de janeiro de 2008 a junho de 2013, foram abatidos 1.305 .723 suínos em 29 abatedouros sob supervisão do SISP, dos quais apenas $125(0,01 \%)$ carcaças apresentaram cisticercose. Do total de carcaças parasitadas, 116 (92,80\%) apresentaram cisticercos calcificados enquanto as demais 9 $(7,20 \%)$ apresentavam-se vivos, conforme pode ser observado na Tabela 1.

Tabela 1: Total e percentual de animais infectados com cisticercose suína, nas formas viva e calcificada, durante os anos de 2008 a 2013 em 29 abatedouros do Estado de São Paulo, Brasil

\begin{tabular}{|c|c|c|c|c|c|}
\hline Ano & $\begin{array}{l}\text { Abatedouros } \\
\text { (n) }\end{array}$ & Animais & $\begin{array}{c}\text { Infectados } \\
\text { n (\%) }\end{array}$ & $\begin{array}{l}\text { Vivos } \\
\mathrm{n}(\%)\end{array}$ & $\begin{array}{c}\text { Calcificados } \\
\text { n (\%) }\end{array}$ \\
\hline 2008 & 23 & 169.071 & $\begin{array}{c}10 \\
(\mathbf{0 , 0 0 5 )}\end{array}$ & $\begin{array}{c}4 \\
(40)\end{array}$ & $\begin{array}{c}6 \\
(60)\end{array}$ \\
\hline 2009 & 21 & 175.913 & $\begin{array}{c}0 \\
(0)\end{array}$ & $\begin{array}{c}0 \\
(0)\end{array}$ & $\begin{array}{c}0 \\
(0)\end{array}$ \\
\hline 2010 & 19 & 225.991 & $\begin{array}{c}33 \\
(0,01)\end{array}$ & $\begin{array}{c}3 \\
(9,1)\end{array}$ & $\begin{array}{c}30 \\
(90,9)\end{array}$ \\
\hline 2011 & 24 & 282.541 & $\begin{array}{c}25 \\
(\mathbf{0 , 0 0 8 )}\end{array}$ & $\begin{array}{c}1 \\
(4)\end{array}$ & $\begin{array}{c}24 \\
(96)\end{array}$ \\
\hline 2012 & 24 & 303.180 & $\begin{array}{c}55 \\
(\mathbf{0}, \mathbf{0 1})\end{array}$ & $\begin{array}{c}0 \\
(0)\end{array}$ & $\begin{array}{c}55 \\
(100)\end{array}$ \\
\hline 2013 & 22 & 149.027 & $\begin{array}{c}2 \\
(0,001)\end{array}$ & $\begin{array}{c}1 \\
(50)\end{array}$ & $\begin{array}{c}1 \\
(50)\end{array}$ \\
\hline Total & & 1.305 .723 & $\begin{array}{c}125 \\
(\mathbf{0}, \mathbf{0 1})\end{array}$ & $\begin{array}{c}9 \\
(7,2)\end{array}$ & $\begin{array}{c}116 \\
(\mathbf{9 2 , 8 )}\end{array}$ \\
\hline
\end{tabular}

Através do baixo número de carcaças parasitadas pode-se supor que as práticas preventivas para a ocorrência da cisticercose estão sendo corretamente realizadas durante a fase de criação desses animais, como também observado no trabalho de Falavigna-Guilherme et al. (2006), que não observaram a ocorrência da cisticercose suína entre os 1.046 suínos abatidos.

Destaca-se que essa cadeia produtiva foi muito modificada em um curto período de tempo, passando de criações extensivas, ou de fundo de quintal, para uma cadeia integrada em todos os elos, do produtor ao abatedouro (Talamini et al., 2005).

No Brasil, a criação de suínos é realizada em sistemas intensivos, que recebem orientação técnica adequada na maioria das propriedades, possuem proprietários instruídos com escolaridade, e que na maioria dos casos se dedicam unicamente a criação dessa espécie. Ainda, durante a criação desses animais estão presentes medidas de biossegurança, práticas sanitárias preventivas e controle sanitário adequados, devido ao grau de desenvolvimento observado nessa cadeia produtiva ao longo dos últimos anos (Embrapa, 2013).

Essa mesma tecnificação na cadeia produtiva da carne suína foi realizada em países da União Europeia, sendo responsável pela eliminação da cisticercose nos plantéis suínos. Porém, nesses países ainda é observada a ocorrência da enfermidade nos rebanhos bovinos, possivelmente pelos modelos produtivos utilizados durante a criação dos animais que utilizam práticas sanitárias inadequadas (Porny et al., 2012).

No presente estudo, durante o mesmo período, foram abatidos 1.434.445 bovinos em 40 estabelecimentos do Estado de São Paulo, dos quais $24.278(1,69 \%)$ carcaças possuíam cisticercos. Desse total de carcaças infectadas, $19.826(81,67 \%)$ estavam na forma calcificada enquanto os demais $4.452(18,33 \%)$ na forma viva. A ocorrência da cisticercose bovina nos 40 abatedouros avaliados durante o período de 2008 a 2013 pode ser observada na Tabela 2.

Pode-se observar uma redução da ocorrência de cisticercos em abatedouros de bovinos durante o período avaliado, no Estado de São Paulo. No ano de 2008 atingiu ocorrência de 3,02\%, enquanto em 2013 foi observado $1,18 \%$ de ocorrência nas carcaças inspecionadas, ou seja, uma redução de 1,84\% durante o período. O aumento observado entre os anos 2012 e 2013 pode ser devido à não disponibilização completa dos dados do ano de 2013 até o presente momento, sendo utilizados apenas os dados de abate do período de seis meses.

A Figura 1 representa os dados da análise de regressão linear da ocorrência mensal de cisticercose bovina ao longo do período avaliado.

A equação da reta mostra que a taxa de ocorrência diminui ao longo do tempo, indicando haver variação de longo prazo, obtendo-se coeficiente de regressão de $-0,0416$, demonstrando haver uma relação inversa entre as variáveis tempo e ocorrência de cisticercose bovina, com tendência de redução. Tal modelo observado na Figura 1 explica $76,03 \%$ da variação observada $\left(R^{2}=\right.$ $0,7603)$. Ainda, utilizando o Software $R^{\circledR}$, encontrou-se que o erro padrão correspondia ao valor de 0,002923, que permite estabelecer o IC 95\%: -0,0047375 a $-0,0359162$, demonstrando uma relação inversa entre as variáveis tempo e taxa $(P<0,05)$.

Tal observação demonstra que a ocorrência de cisticercose em carcaças bovinas sofreu diminuição ao longo do período avaliado potencialmente devido a melhorias em práticas preventivas utilizadas nas etapas de criação dos animais, conforme avaliado por Deschamps et al. (2013).

Ungar e Germano (1992) verificaram frequência de 5,5\% de cisticercose bovina, no ano de 1986, durante o abate em 47 estabelecimentos sob Serviço de Inspeção Federal (SIF) localizados no Estado de São Paulo. Também foi realizado outro estudo envolvendo 1.976 .824 bovinos abatidos no período de 1990 a 2000 , em frigoríficos com SIF na região de Araçatuba, revelando ocorrência de $4,18 \%$ nas carcaças inspecionadas (Fernandes e Buzetti, 2001). Ainda, Fukuda et al.(2003), 
Tabela 2: Total e percentual de animais infectados com cisticercose bovina, nas formas viva e calcificada, durante os anos de 2008 a 2013 em 40 abatedouros do Estado de São Paulo, Brasil

\begin{tabular}{|c|c|c|c|c|c|}
\hline Ano & $\begin{array}{l}\text { Abatedouros } \\
\text { (n) }\end{array}$ & Animais & $\begin{array}{c}\text { Infectados } \\
\text { n (\%) }\end{array}$ & $\begin{array}{l}\text { Vivos } \\
\mathrm{n}(\%)\end{array}$ & $\begin{array}{c}\text { Calcificados } \\
\text { n (\%) }\end{array}$ \\
\hline 2008 & 31 & 171.753 & $\begin{array}{l}5.198 \\
(3,02)\end{array}$ & $\begin{array}{c}887 \\
(17,07)\end{array}$ & $\begin{array}{c}4.311 \\
(82,93)\end{array}$ \\
\hline 2009 & 27 & 145.648 & $\begin{array}{l}4.082 \\
(2,80)\end{array}$ & $\begin{array}{c}500 \\
(\mathbf{1 2 , 2 4 )}\end{array}$ & $\begin{array}{c}3.582 \\
(87,76)\end{array}$ \\
\hline 2010 & 27 & 256.275 & $\begin{array}{l}4.940 \\
(1,92)\end{array}$ & $\begin{array}{c}869 \\
(17,60)\end{array}$ & $\begin{array}{c}4.071 \\
(82,40)\end{array}$ \\
\hline 2011 & 32 & 277.474 & $\begin{array}{l}3.921 \\
(\mathbf{1}, \mathbf{4 1})\end{array}$ & $\begin{array}{c}847 \\
(21,60)\end{array}$ & $\begin{array}{c}3.074 \\
(78,40)\end{array}$ \\
\hline 2012 & 32 & 383.758 & $\begin{array}{l}3.770 \\
(0,98)\end{array}$ & $\begin{array}{c}925 \\
(\mathbf{2 4 , 2 5 )}\end{array}$ & $\begin{array}{c}2.845 \\
(\mathbf{7 5}, 45)\end{array}$ \\
\hline 2013 & 31 & 199.537 & $\begin{array}{l}2.367 \\
(1,18)\end{array}$ & $\begin{array}{c}424 \\
(17,91)\end{array}$ & $\begin{array}{c}1.943 \\
(\mathbf{8 2 , 0 9 )}\end{array}$ \\
\hline Total & & 1.434 .445 & $\begin{array}{c}24.278 \\
(1,69)\end{array}$ & $\begin{array}{c}4.452 \\
(18,33)\end{array}$ & $\begin{array}{l}19.826 \\
(\mathbf{8 1 , 6 7 )}\end{array}$ \\
\hline
\end{tabular}

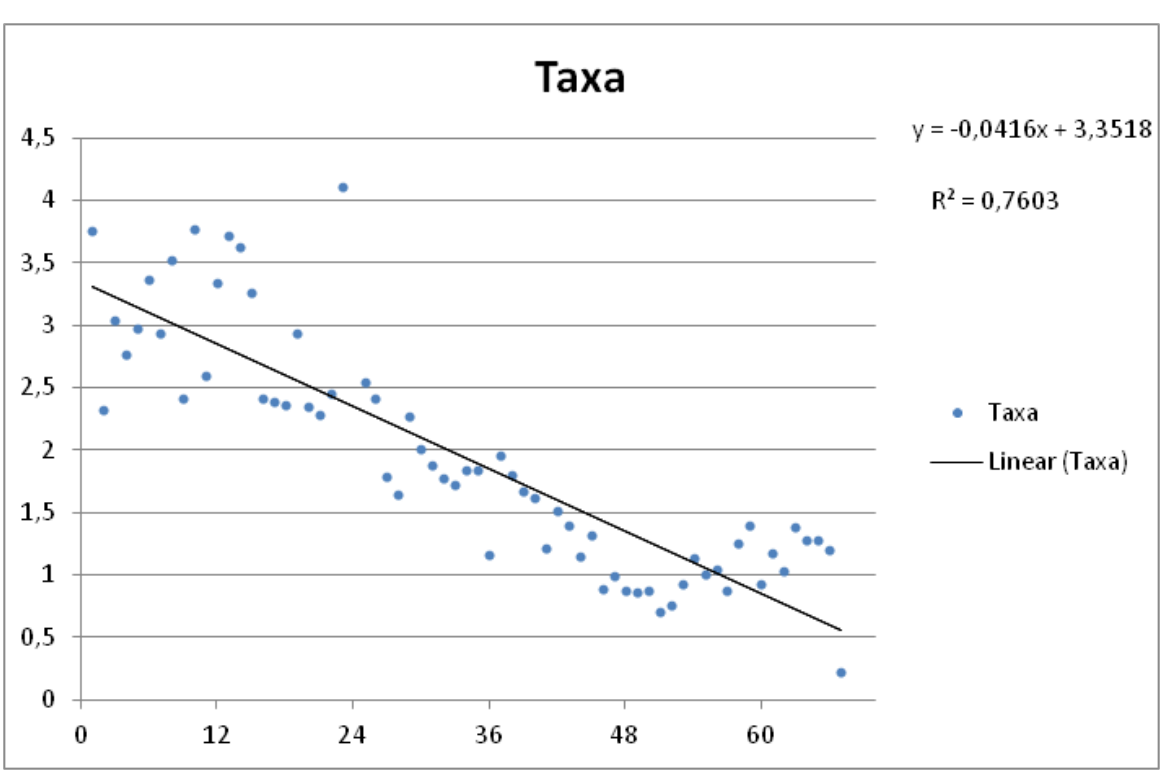

Figura 1: Regressão linear da ocorrência de cisticercose bovina (eixo $Y$ ) durante os meses de janeiro de 2008 a junho de 2013 (eixo X) em 40 abatedouros do Estado de São Paulo, Brasil. abatedouros entre os anos de 1999 e 2001. Já em outro estudo desenvolvido em matadouro-frigorífico sob SIF localizado no Município de Sertãozinho/SP, durante os anos de 2000 a 2002, foi verificada frequência de cisticercose de 2,9\% nos bovinos abatidos (RezendeLago et al., 2011). Novamente os valores observados nesses estudos são superiores ao valor encontrado no presente trabalho, demonstrando a redução na ocorrência da cisticercose bovina.

Apesar de ser observada uma redução da cisticercose bovina ao longo do período, essa ainda ocorre em níveis que acarretam importantes perdas econômicas aos pecuaristas e às indústrias, necessitando que sua redução de ocorrência atinja pequenos níveis, igualmente aos observados na cadeia produtiva da carne suína brasileira.

Santos et al. (2013) avaliaram o perfil epidemiológico da cisticercose bovina no Município de Viçosa, Estado de Minas Gerais, e verificaram a presença de fatores de risco para a manutenção da enfermidade, destacando as práticas de fornecimento de água sem tratamento aos animais, criação de animais para serem abatidos clandestinamente, criação dos bovinos com baixa adoção de tecnologia como possíveis fatores. Todas essas práticas devem ser evitadas em programas de BPA, demonstrando a necessidade da implantação das mesmas na pecuária bovina brasileira (Valle, 2011) para que a enfermidade seja controlada igualmente na espécie suína.

A adoção das BPA como ferramenta preventiva para a ocorrência de cisticercose bovina também foi demonstrada por Deschamps et al., (2013) e Rossi et al.(2015). Os autores avaliaram a ocorrência de cisticercose bovina na França em propriedades fornecedoras de bovinos para o abate e determinaram que a ausência de adesão a programas de auditorias e certificação internacional atua como fator de risco para ocorrência da enfermidade. A certificação das propriedades mostrou ser um fator de redução de risco para a ocorrência da enfermidade pois consiste em uma adequada adoção e correto monitoramento das práticas utilizadas nas propriedades para atender às exigências sanitárias exigidas. utilizando os dados de todos os frigoríficos sob SIF no Estado de São Paulo, evidenciaram frequência de $4,28 \%$ no período de 1980 a 2001 . Esses valores observados foram superiores ao observado no presente estudo, demonstrando melhorias na prevenção da enfermidade no rebanho bovino do Estado de São Paulo.

Ainda no mesmo estado, Manhoso e Prata (2004) observaram prevalência média de $9,37 \%$ de cisticercose bovina em

\section{Conclusão}

A ocorrência de cisticercose bovina é superior à observada nos suínos abatidos, com redução significativa ao longo do período de 2008 a 2013. São necessárias melhorias na adoção de práticas sanitárias preventivas como os programas de Boas Práticas Agropecuárias (BPA) durante a criação de bovinos para reduzir a ocorrência da cisticercose nessa espécie igualmente é observada em suínos. 


\section{Referências}

BRASIL, Ministério da Agricultura, Pecuária e Abastecimento Regulamento da Inspeção Industrial e Sanitária dos Produtos de Origem Animal (RIISPOA), Brasília, 1952.

DESCHAMPS, J.; CALAVAS,D.; MIALET, S.; GAY, E.; DUPUY, C. A preliminary investigation of farm-level risk factors for cattle condemnation at the slaughterhouse: A case control study on French farms. Preventive Veterinary Medicine,; v. 112, n. 3-4, p .428-432, 2013.

EMBRAPA. Empresa Brasileira de Pesquisa Agropecuária. Caracterização da suinocultura no Brasil a partir do censo agropecuário 2006 do IBGE. Documentos 160. 2013. Concórdia (SC). Disponível em: www.cnpsa.embrapa.br/sgc/sgc publicacoes/publicacao_c9l46g5m.pdf, Acesso em 05 out. 2014.

FALAVIGNA-GUILHERME, A.L.; SILVA, K.; ARAÚJO, S.M.; TOBIAS, M.L.; FALAVIGNA, D.M.L. Cisticercose em animais abatidos em Sabáudia, Estado do Paraná. Arquivo Brasileiro de Medicina Veterinária e Zootecnia, v. 58, n. 5, p. 950-951, 2006.

FERNANDES, J.O.M.; BUZETTI, W.A.S. Prevalência de cisticercose bovina em animais abatidos em frigoríficos sob inspeção federal, da $9^{a}$ região administrativa de Araçatuba, SP. Revista. Higiene Alimentar, v.15, n. 87, p. 30-37, 2001.

FUKUDA, R.T.; PRATA, L.F.; VERARDINO, H.; ALMEIDA, L.A.M. Evolução da cisticercose bovina em animais abatidos no Estado de São Paulo. Revista Higiene Alimentar, v.17, n.108, p. 21-31, 2003.

MANHOSO, F.F.R., PRATA, L.F. Prevalência da cisticercose bovina na região oeste do Estado de São Paulo. Revista Higiene Alimentar, v. 18, n. 121, p. 42-49, 2004.

MEDEIROS, E.S.; OLIVEIRA, J.T.F. DE; PORTO, W.J.N.; PINHEIRO JÚNIOR, J. W.; SOARES, K.D.A.; ALVES, L.C. Prevalência da cisticercose em um matadouro sob inspeção federal no Município de Promissão no Estado de São Paulo. In: V Congresso Latino Americano e XI Congresso de Higienistas de Alimentos, Salvador, Bahia. 2011. Anais. Disponível em: <http:// www.sovergs.com.br/site/higienistas/trabalhos/10363.pdf>, Acesso em 02 out. 2014.

PFUETZENREITER, M.R.; ÁVILA-PIRES, F.D. Epidemiologia da teníase/cisticercose por Taenia soliume Taenia saginata. Ciência Rural, v. 30, n. 3, p. 541-548, 2000.
PINTO, P.S.A. Inspeção e Higiene de Carnes. Viçosa: Editora UFV, 2008, p. 269- 274.

PORNY, P.; PRAET, N. GABRIEL, S. Cysticercosys and meat safety in the UE - status and perspectives. Biological Food Safety \& Quality, Proceedings of the Internacional Conference.Belgrade, Serbia, p. 32-34, 2012.Disponível em: http://www.cabi.org/ cabdirect/FullTextPDF/2013/20133110010.pdf

ROSSI, G.A.M.; HOPPE, E.G.L.; MATHIAS L.A.; VIDALMARTINS, A.M.C.; MUSSI, L.A.; PRATA, L. F. Bovine cysticercosis in slaughtered cattle as an indicator of Good Agricultural Practices (GAP) and epidemiological risk factors. Preventive Veterinary Medicine, v.118, n. 4, p. 504-508, 2015

REZENDE-LAGO, N.C.M.; REIS, L.S. DOS; MARCHI, P.G.F. $D E$. Levantamento epidemiológico da cisticercose e tuberculose em bovinos abatidos sob inspeção federal no município de Sertãozinho, SP. Revista Higiene Alimentar, v. 25, n. 192/193, p.175-181, 2011.

SANTOS, J.M.G.; BARROS, M.C.R.B. Endoparasitas de Importância no Comércio da Carne, Revista Agronegócio e Meio Ambiente, v. 2, n. 1, p. 21-39, 2009.

SANTOS, T.O.; PINTO, P.S.A.; IASBIK, A.F.; SSILVA, L.F.; NIETO, E.C.A.; GUIMARÃES-PEIXOTO, R.P.M. Epidemiological survey of the taeniasis/cysticercosis complex in cattle farms in Viçosa County, Minas Gerais, Brazil. Pesquisa Veterinária Brasileira, v. 33, n. 4, p. 499-452, 2013.

TALAMINI, E.; PEDROZZO, E.A.; SILVA, A.L. Gestão da cadeia de suprimentos e a segurança do alimento: uma pesquisa exploratória na cadeia exportadora da carne suína. Gestão \& Produção, 2005; v. 12, n. 1, p. 107-120. Disponível em: http:// www.scielo.br/pdf/gp/v12n1/a10v12n1.pdf, Acesso em 05 out. 2014.

UNGAR, M.L.; GERMANO, P.M.L. Prevalência da cisticercose bovina no Estado de São Paulo. Revista de Saúde Pública, v. 26, n. 3, p. 167-172, 1992.

VALLE, E.R do. Embrapa Gago de Corte. Manual de orientações: BrazilianGap- Boas práticas agropecuárias - Bovinos de corte, Embrapa 2ed, 2011. Disponívelem: http://cloud.cnpgc.embrapa. br/bpa/files/2013/02/MANUAL_de-BPA_NACIONAL.pdf Acesso em: 5. jun. 2013. 